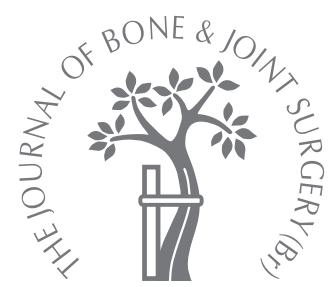

\title{
Return to theatre following total hip and knee replacement, before and after the introduction of rivaroxaban
}

\author{
A RETROSPECTIVE COHORT STUDY
}

C. D. Jensen,

A. Steval,

P. F. Partington,

M. R. Reed,

S. D. Muller

From Wansbeck

General Hospital,

Northumberland,

United Kingdom
- C. D. Jensen, MBBS, MRCS, Specialist Registrar(Tr \& Orth) I. P. Partington, $\mathrm{FRCS}(\operatorname{Tr} \&$ Orth), Consultant Orthopaedic Surgeon

M. R. Reed, MD, FRCS $(T r$ \& Orth), Consultant Orthopaedic Surgeon

S. D. Muller, MD, FRCS(Tr \& Orth), Consultant Orthopaedic Surgeon

Wansbeck General Hospital, Woodhorn Lane, Ashington, Northumberland NE63 9JJ, UK.

A. Steval, Final Year Medical Student

Medical School, University of Newcastle upon Tyne,

Framlington Place, Newcastle NE2 4HH, UK.

Correspondence should be sent to $\mathrm{Mr}$ C. D. Jensen; e-mail: jensen@doctors.org.uk

(C)2011 British Editorial Society of Bone and Joint Surgery doi:10.1302/0301-620X.93B1. $24987 \$ 2.00$

$J$ Bone Joint Surg [Br] 2011;93-B:91-5.

Received 7 April 2010; Accepted after revision 5 October 2010

Rivaroxaban has been recommended for routine use as a thromboprophylactic agent in patients undergoing lower-limb arthroplasty. However, trials supporting its use have not fully evaluated the risks of wound complications. This study of 1048 total hip/knee replacements records the rates of return to theatre and infection before and after the change from a low molecular weight heparin (tinzaparin) to rivaroxaban as the agent of chemical thromboprophylaxis in patients undergoing lower-limb arthroplasty. During a period of 13 months, 489 consecutive patients undergoing lower-limb arthroplasty received tinzaparin and the next 559 consecutive patients received rivaroxaban as thromboprophylaxis.

Nine patients in the control (tinzaparin) group (1.8\%, 95\% confidence interval 0.9 to 3.5 ) returned to theatre with wound complications within 30 days, compared with 22 patients in the rivaroxaban group $(3.94 \%, 95 \%$ confidence interval 2.6 to 5.9$)$. This increase was statistically significant $(p=0.046)$. The proportion of patients who returned to theatre and became infected remained similar $(p=0.10)$.

Our study demonstrates the need for further randomised controlled clinical trials to be conducted to assess the safety and efficacy of rivaroxaban in clinical practice, focusing on the surgical complications as well as the potential prevention of venous thromboembolism.

Rivaroxaban (Xarelto; Bayer Schering Pharma AG, Wuppertal, Germany) is one of the first oral factor $\mathrm{Xa}$ inhibitors to be licensed for thromboprophylaxis after total knee and hip replacement surgery. Four large studies conducted by RECORD (The Regulation of Coagulation in Orthopaedic Surgery to Prevent Deep Vein Thrombosis and Pulmonary Embolism) have shown it to be effective in preventing venous thromboembolism compared with enoxaparin (Clexane/Lovenox; Sanofi-Aventis, Frankfurt, Germany). ${ }^{1-4}$ These studies were the sole evidence on which the National Institute of Clinical Excellence (NICE) ${ }^{5}$ based the approval of rivaroxaban for use as chemical thromboprophylaxis following hip and knee joint replacement in England and Wales. Although these studies demonstrated no significant increase in the rates of major bleeding, concerns have been raised about the lack of data on other potential surgical complications. ${ }^{6-20}$ Surgical outcomes such as the rate of wound healing, haematoma formation and drainage were either not included or only addressed as secondary safety outcomes by the RECORD trials. These complications have previously been shown to increase rates of wound infection and subsequent return to theatre. ${ }^{12,13}$ Risk factors for developing a wound complication and/or infection after a total hip (THR) or knee replacement (TKR) include thromboprophylaxis, immunosuppressive therapy, prolonged wound drainage, obesity, diabetes mellitus, hypothyroidism, renal failure and previous open surgical procedures. $^{12,13}$

Following concerns raised by an author from the RECORD 4 group, ${ }^{11}$ this study aimed to report the effects of rivaroxaban on wound complications, infections and return to theatre in patients undergoing THR and TKR.

\section{Patients and Methods}

Between February 2009 and February 2010, all the patients who underwent a THR or TKR at our hospital and returned to theatre with a wound-related complication within 30 days of their operation were included in the study. The study period of 13 months included six months prior to and seven months following the introduction of rivaroxaban as the agent of choice for chemical thromboprophylaxis.

Group 1 comprised patients who had their primary operation between 1 February 2009 and 31 July 2009 (six months). These patients 
Table I. Demographics and risk factors for wound complications in return to theatre patients in the two groups

\begin{tabular}{|c|c|c|c|}
\hline & $\begin{array}{l}\text { Group } 1 \text { (tinzaparin) } \\
(\mathrm{n}=9)\end{array}$ & $\begin{array}{l}\text { Group } 2 \text { (rivaroxaban) } \\
(\mathrm{n}=22)\end{array}$ & $\begin{array}{l}\text { Statistical difference } \\
\text { between groups } 1 \text { and } 2\end{array}$ \\
\hline \multicolumn{4}{|l|}{ Gender } \\
\hline $\mathrm{M}: \mathrm{F}$ & $5: 4$ & $8: 14$ & $0.43^{\dagger}$ \\
\hline Operation ratio (TKR:THR) ${ }^{*}$ & $1: 8$ & $6: 16$ & $0.64^{\dagger}$ \\
\hline Mean age in years (range) & 67 (44 to 81$)$ & 64 (39 to 86 ) & 0.93 \\
\hline Body mass index $\left(\mathrm{kg} / \mathrm{m}^{2}\right)$ (range) & 31 (26 to 36$)$ & 31 (23 to 41$)$ & $1.00^{\ddagger}$ \\
\hline Chronic renal failure (\%) & $5(55)$ & $7(30)$ & $0.25^{\dagger}$ \\
\hline Diabetes (\%) & $1(11)$ & $2(9)$ & $1.00^{\dagger}$ \\
\hline Hypothyroidism (\%) & $1(11)$ & $3(13)$ & $1.00^{\dagger}$ \\
\hline Immunosuppressive medication (\%) & $0(0)$ & $1(4)$ & $1.00^{\dagger}$ \\
\hline Antithrombotic medication (\%) & $3(33)$ & $7(30)$ & $1.00^{\dagger}$ \\
\hline
\end{tabular}

received tinzaparin (Innohep; LEO Pharma A/S, Ballerup, Denmark) (4500 U subcutaneously, once daily) as thromboprophylaxis from day one post-operatively for 28 days, in accordance with the hospital protocol, which was based on the NICE guidelines.

Group 2 had their primary operation between 1 August 2009 and 28 February 2010 (seven months). They received rivaroxaban (10 mg orally, once daily) as thromboprophylaxis from day one post-operatively in accordance with the new hospital protocol, which reflects the latest guidance from NICE. THRs received prophylaxis for 28 days and TKRs for 14 days.

Patients in both of the groups also wore thromboembolic deterrent stockings for six weeks after surgery, received the same single intravenous dose of prophylactic antibiotics, and were encouraged to mobilise early in the post-operative period. No drains were used at the operation in either group.

Theatre logs from the study period were analysed to identify all patients who had returned to theatre within 30 days of the operation. The hospital case notes were analysed to record demographics and relevant comorbidities that might have contributed as risk factors for delayed wound healing, post-operative bleeding or wound infection (Table I).

Patients were excluded from the return to theatre subanalysis groups if the indication for this was not related to the wound. In group 1, one patient was excluded because of dislocation. In group 2, one patient was excluded because of wound dehiscence after a fall at home, and two were excluded because of dislocation (Fig. 1).

Return to theatre for a wound-related complication was defined as returning to theatre for open irrigation and debridement of a wound within 30 days of the operation. The indication for surgical management of wound problems remained unchanged during the period of the study and was at the clinical discretion of the nine consultant surgeons involved. Indications for return to theatre included clinical signs of wound infection and/or haematoma and raised inflammatory blood markers (including rising trends in CRP, ESR and white cell count). As this study was not a

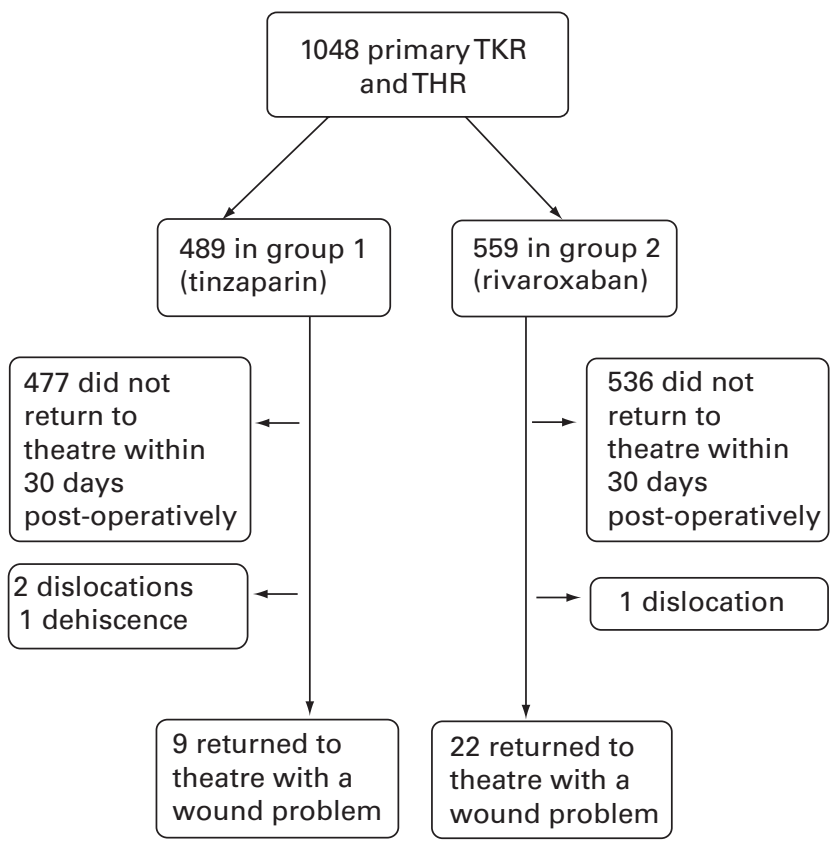

Fig. 1

Study design showing the consort flowchart (TKR, total knee replacement; THR, total hip replacement).

prospective trial, fixed criteria and protocols for indications for return to theatre could not be used. Clinical signs of infection and rising inflammatory markers informed the decisions of the consultant surgeons involved. All the patients who returned to theatre with wound complications had microbiological specimens taken (fluid and tissues) according to departmental protocol. Standard sampling techniques were used, with five samples taken and sterile instruments used for each. The bacterial culture results were retrieved from the hospital laboratory database. The wound was considered deeply infected if the primary samples taken from deep tissues isolated the same organism, after standard and enriched culture. 
Statistical analysis. Analysis was on an intention-to-treat basis. All statistical analyses were performed using SPSS version 14.0 (SPSS Inc., Chicago, Illinois). Categorical data were analysed using a $2 \times 2$ contingency table with chisquared and Fisher's exact tests as dictated by sample size. Continuous data were analysed using the Student's $t$-test. The null hypothesis was that there would be no difference in the return to theatre rates between group 1 and group 2 . Statistical significance would be reached at a confidence interval $(\mathrm{CI})$ of $95 \%(\mathrm{p}<0.05)$.

\section{Results}

There were a total of 1048 patients in the study: 489 in group 1 and 559 in group 2. There were a similar proportion of THRs and TKRs performed in each group: 195:294 and 249:310, respectively (chi-squared test, $\mathrm{p}=0.13)$.

In group 1 (tinzaparin), nine of the 489 patients $(1.8 \%$, $95 \%$ CI 0.9 to 3.5$)$ returned to theatre with wound complications. In group 2 (rivaroxaban), 22 of 559 patients returned to theatre $(3.94 \%, 95 \%$ CI 2.6 to 5.9$)$. This increase was statistically significant $(\mathrm{p}=0.046)$. Demographics and comorbidities were similar in the subgroups of return to theatre patients (Table I).

Of those patients who returned to theatre, microbiology results showed that five of the nine $(55.5 \%)$ in group 1 had a deep infection, compared with 14 of $22(63.6 \%)$ in group $2(\mathrm{p}=0.7)$. The overall rate of deep infection in group 1 was $1 \%$ (95\% CI 0.4 to 2.4 ), compared with $2.5 \%$ (95\% CI 1.5 to 4.2 ) in group 2 ( $\mathrm{p}=0.102)$.

Rates of venous thromboembolism remained similar in both groups. The incidence of symptomatic radiologically confirmed pulmonary embolism was $0.8 \%$ (95\% CI 0.24 to 2.16 ) in group 1 and $0.9 \%$ (95\% CI 0.32 to 2.14 ) in group $2(\mathrm{p}=1.00)$.

Analysis of the return to theatre patients (Table II) revealed that there was no statistically significant difference in the mean time from primary operation to return to theatre between groups 1 and 2 (17.22 days ( 8 to 25$) v s$ 16.81 days ( 6 to 30$), p=0.89$ ). Wound haematoma was the stated indication for return to theatre in significantly more cases after rivaroxaban was introduced ( $0 v s 9, \mathrm{p}=0.032)$. The return to theatre patients were more likely to require more than one washout ( $3 v s 9, \mathrm{p}=1.00)$ and a total joint revision (1 vs 2, $\mathrm{p}=1.00)$ after rivaroxaban was introduced, but neither observation was statistically significant. The analysis of this subgroup based on THRs and TKRs separately is shown in Table III.

The mean length of hospital stay for the primary operation in patients who subsequently returned to theatre in group 1 (tinzaparin) was 6.1 days (2 to 8 ), and in group 2 (rivaroxaban) was six days (2 to 10 ). There was no statistically significant difference in the mean total length of stay for those patients who returned to theatre in groups 1 and 2 (21.0 days (10 to 32 ) vs 18.8 days ( 7 to 79 ), $95 \%$ CI -9.22 to $13.58, \mathrm{p}=0.70)$.

\section{Discussion}

Controversy exists over the routine use of chemical thromboprophylaxis in lower-limb arthroplasty. In 2007, 2009 and 2010 NICE published guidelines recommending extended-duration chemical thromboprophylaxis for all patients undergoing lower-limb arthroplasty within the NHS in England and Wales. ${ }^{14}$ Subsequent correlation of the National Joint Registry (England and Wales) and the Hospital Episode Statistics ${ }^{15}$ data has shown an increased rate of venous thromboembolism in patients undergoing THR despite an increased use of low molecular weight heparin chemical thromboprophylaxis. Additional concern exists regarding an increase in the incidence of complications associated with chemical thromboprophylaxis, such as prolonged wound drainage ${ }^{12}$ and thrombocytopenia. ${ }^{15,16}$

The RECORD trials were deficient in their lack of measurement of surgical outcomes such as wound healing, drainage, infection, range of movement and chronic pain. This led one of the authors of the RECORD4 paper ${ }^{2}$ to later state that he would not recommend it (rivaroxaban) for his patients. ${ }^{10}$

Prolonged wound drainage after lower-limb arthroplasty is associated with infection, longer hospital stay, reoperation, and a subsequent increase in the economic burden on the national resources. ${ }^{17}$ We could not find any reports focusing on the potential wound complications associated with the use of oral factor $\mathrm{Xa}$ inhibitors such as rivaroxaban.

In this study period, patients who received rivaroxaban were more than twice as likely to return to theatre with a wound complication after THR or TKR as those who received tinzaparin $(3.94 \%$ vs $1.8 \%, \mathrm{p}=0.046)$. In both groups, patients having a THR were more likely to return to theatre compared with those having a TKR (group 1, 8 vs $1, \mathrm{p}=0.004$, and group 2, 16 vs 6, chi-squared test, $\mathrm{p}=0.015$ ) (Table III).

Separating the TKR from the THR patients still shows an increase in the rate of return to theatre after changing from tinzaparin to rivaroxaban as the thromboprophylactic agent. In the TKR patients the rate increased from $0.3 \%$ ( 1 of 294 ) to $2 \%$ (6 of 249 ) after the change to rivaroxaban. This was statistically significant $(\mathrm{p}=0.05)$. In the THR patients the rate increased from $4.1 \%$ (8 of 195 ) to $5.2 \%$ (16 of 310$)$, but this did not reach statistical significance $(\mathrm{p}=0.67)$ (Table III).

Post-operative wound infection remains a major burden on both the patient and the healthcare provider. Deep infection following arthroplasty is a high-morbidity complication that often requires many surgical debridements, protracted courses of expensive and potentially toxic antibiotics, prolonged hospital stay with immobilisation and isolation, and frequently staged revision procedures. ${ }^{21}$ Our rate of infection increased from $1 \%$ to $2.5 \%$ following the introduction of rivaroxaban. An infection rate of $1 \%$ is similar to that reported in the literature following hip and knee replacement. ${ }^{22,23}$ 
Table II. Further analysis of return to theatre (RTT) patients: nine in the tinzaparin group (T1 to 9) and 22 in the rivaroxaban group (R1 to 22)

\begin{tabular}{|c|c|c|c|c|c|c|c|}
\hline Patient & Primary operation ${ }^{*}$ & RTT indication & $\begin{array}{l}\text { Time to RTT } \\
\text { (days) }\end{array}$ & Infection & Organism & $\begin{array}{l}\text { > } 1 \text { RTT } \\
\text { (washout) }\end{array}$ & Revision \\
\hline T1 & THR & Wound ooze & 20 & No & & No & No \\
\hline T2 & THR & Wound ooze & 8 & No & & No & No \\
\hline T3 & THR & Wound ooze & 11 & No & & No & No \\
\hline T4 & THR & Wound ooze & 15 & Yes & S. epidermidis (resistant) & Yes & Yes \\
\hline T5 & THR & Wound ooze & 25 & Yes & Staph. aureus & Yes & No \\
\hline T6 & THR & Wound ooze & 16 & Yes & Staph. aureus & Yes & No \\
\hline T7 & TKR & Wound ooze & 18 & Yes & Staph. aureus & No & No \\
\hline T8 & THR & Wound ooze & 22 & Yes & $\begin{array}{l}\text { Coagulase-negative } \\
\text { staphylococcus }\end{array}$ & $\begin{array}{l}\text { Patient } \\
\text { refused }\end{array}$ & No \\
\hline T9 & THR & Wound infection & 20 & No & & No & No \\
\hline R1 & TKR & Wound ooze & 29 & Yes & Klebsiella sp. & Yes & No \\
\hline $\mathrm{R} 2$ & THR & Wound ooze & 11 & Yes & S. epidermidis & Yes & No \\
\hline R3 & THR & Discharging haematoma & 7 & No & & No & No \\
\hline R4 & TKR & Wound infection & 30 & No & & No & No \\
\hline R5 & THR & Discharging haematoma & 10 & No & & No & No \\
\hline R6 & TKR & Wound ooze & 23 & Yes & Coliforms & No & No \\
\hline R7 & THR & Discharging haematoma & 13 & Yes & S. epidermidis & No & No \\
\hline R8 & THR & Wound ooze & 16 & Yes & S. epidermidis & No & No \\
\hline R9 & THR & Wound ooze & 23 & No & & Yes & No \\
\hline R10 & THR & Discharging haematoma & 8 & No & & Yes & No \\
\hline R11 & THR & Wound ooze & 14 & No & & No & No \\
\hline R12 & THR & Wound ooze & 6 & No & & No & No \\
\hline R13 & TKR & Discharging haematoma & 30 & Yes & E. coli & Yes & No \\
\hline R14 & TKR & Discharging haematoma & 14 & Yes & E. coli & No & No \\
\hline R15 & THR & Wound ooze & 26 & Yes & S. epidermidis & Yes & No \\
\hline R16 & TKR & Discharging haematoma & 21 & Yes & $\mathrm{MRSA}^{\dagger}$ & Yes & No \\
\hline R17 & THR & Wound ooze & 30 & Yes & Staph. aureus & Yes & Yes \\
\hline R18 & THR & Discharging haematoma & 10 & Yes & Staph. aureus & No & No \\
\hline R19 & THR & Wound ooze & 9 & Yes & S. epidermidis & No & No \\
\hline $\mathrm{R} 20$ & THR & Wound ooze & 14 & Yes & S. epidermidis & Yes & Yes \\
\hline R21 & THR & Discharging haematoma & 13 & Yes & Coliforms & Yes & No \\
\hline $\mathrm{R} 22$ & THR & Wound ooze & 13 & No & & No & No \\
\hline
\end{tabular}

* THR, total hip replacement; TKR, total knee replacement

† MRSA, methicillin-resistant Staphylococcus aureus

Table III. Analysis of the rates of infection and return to theatre by the type of arthroplasty

\begin{tabular}{llllll}
\hline & \multicolumn{3}{l}{ Tinzaparin } & & \multicolumn{2}{l}{ Rivaroxaban } \\
\cline { 2 - 3 } \cline { 5 - 6 } & THR $^{*}$ (n= 195) & $\mathbf{T K R}^{\dagger}(\mathbf{n}=\mathbf{2 9 4})$ & & THR (n= 310) & TKR (n = 249) \\
\hline Return to theatre (\%) $8(4.1)$ & $1(0.3)$ & $16(5.2)$ & $6(2.4)$ \\
Infection (\%) & $4(2.1)$ & $1(0.3)$ & $9(2.9)$ & $5(2.0)$ \\
\hline
\end{tabular}

* THR, total hip replacement

$\dagger \mathrm{TKR}$, total knee replacement

Our rate of venous thromboembolism events remained unchanged; however, the study size would be underpowered to detect a difference, and no meaningful statistical analysis could therefore be performed.

On further detailed analysis of the return to theatre cases it was noted that the stated indication for this was a 'wound haematoma' in significantly more cases after rivaroxaban was introduced ( 0 vs $9, \mathrm{p}=0.032$ ).

The mean hospital length of stay for a primary TKR or THR in our hospital at the time of this study was 5.3 days (1 to 79). Patients who subsequently had wound complications and returned to theatre were noted to have a slightly longer mean length of stay. It may have been that this was because of concerns over their persistently leaking wounds, but data here are lacking. The mean total length of stay in these patients (including the initial stay for the primary procedure as well as the return to theatre hospital stay) was 22 days ( 7 to 79 ). There was no statistically significant difference in the mean total length of stay between groups 1 and $2(p=0.70)$; however, the observed increase in the number of return to theatre patients in the rivaroxaban group led to an overall increase in the total number of extra hospital days required for this group. 
Several cost-effectiveness models have shown oral anticoagulants, including rivaroxaban, to be more effective and less expensive than enoxaparin sodium; $;^{24,25}$ however, these models fail to take into account the risk of prolonged wound drainage, post-operative infection and haematoma, which are known to have high personal, healthcare and socioeconomic costs.

Our hospital was one of the first in the United Kingdom to introduce rivaroxaban as thromboprophylaxis for all THRs and TKRs. In the RECORD trials, enoxaparin was used as the control drug, ${ }^{1-4}$ however, tinzaparin was used as the control drug in this trial, as this was the established Trust protocol. Direct comparison trials in THR have shown enoxaparin to be of equal efficacy and safety to tinzaparin. ${ }^{26}$

This was a retrospective, non-randomised cohort trial, and therefore cannot causally link the use of rivaroxaban with an increased rate of wound complication; however, the only variable that changed in the study period was the chemical prophylaxis.

The retrospective nature of the study may be considered as a potential weakness, but also a potential strength, as the surgeons' decision was not influenced by an ongoing prospective study. Other weaknesses include a disparate group size and multiple surgeons in the series. The study durations, and hence group sizes for groups 1 and 2 were unequal. Retrospective analysis could not be extended beyond February 2009, as antibiotic prophylaxis changed at this point in a drive to reduce iatrogenic Clostridium difficile colitis associated with cephalosporins. Operations in both groups were carried out by the same nine surgeons, including three authors (PFP, MRR, SDM).

Thromboembolism after arthroplasty is considered to be a potentially serious complication. The incidence of venous thromboembolism events in the practice of modern arthroplasty has been challenged; ${ }^{27}$ however, current NICE guidelines continue to recommend extended chemical prophylaxis.

The risk of venous thromboembolism needs to be balanced against the potential medical and surgical complications of the drug used to prevent it. The RECORD trials do not adequately assess these surgical complications, and focus only on the risk of major bleeding. Our study demonstrates the urgent need for further randomised controlled clinical trials to assess the safety and efficacy of rivaroxaban in clinical practice, focusing on the surgical complications as well as the potential prevention of venous thromboembolism. Based on this study, we have discontinued the use of rivaroxaban in our hospital until robust evidence from independent randomised clinical trials becomes available.

No benefits in any form have been received or will be received from a commer cial party related directly or indirectly to the subject of this article.

\section{References}

1. Kakkar AK, Brenner B, Dahl OE, et al; RECORD2 Investigators. Extended dura tion rivaroxaban versus short-term enoxaparin for the prevention of venous thromboembolism after total hip arthroplasty: a double-blind, randomised controlled trial. Lancet 2008;372:31-9.
2. Turpie AG, Lassen MR, Davidson BL, et al; RECORD4 Investigators. Rivaroxaban versus enoxaparin for thromboprophylaxis after total knee arthroplasty (RECORD4): a randomised trial. Lancet 2009;373:1673-80.

3. Eriksson BI, Borris LC, Friedman RJ, et al; RECORD1 Study Group. Rivaroxaban versus enoxaparin for thromboprophylaxis after hip arthroplasty. $N$ Eng/ J Med 2008;358:2765-75

4. Lassen MR, Ageno W, Borris LC, et al; RECORD3 Investigators. Rivaroxaban versus enoxaparin for thromboprophylaxis after total knee arthroplasty. N Engl J Med 2008;358:2776-86.

5. Atkins RM. NICE and venous thromboembolism. J Bone Joint Surg [Br] 2010;92B:609-10.

6. Van Thiel D, Kalodiki E, Wahi R, et al. Interpretation of benefit-risk of enoxaparin as comparator in the RECORD program: rivaroxaban oral tablets (10 milligrams) for use in prophylaxis in deep vein thrombosis and pulmonary embolism in patients undergoing hip or knee replacement surgery. Clin Appl Thromb Hemost 2009;15:38994.

7. Longo UG, Maffulli N, Denaro V. Rivaroxaban versus enoxaparin after total knee arthroplasty. Lancet 2009;374:681-2.

8. Raju NC, Dimmitt S, Eikelboom JW. Rivaroxaban versus enoxaparin after total knee arthroplasty. Lancet 2009;374:681

9. Stief T. Rivaroxaban versus enoxaparin after total knee arthroplasty. Lancet 2009;374:681

10. Gómez-Outes A, Suárez-Gea ML, Blázquez-Pérez A, Poxo-Hernández C, Vargas-Castrillón E. Rivaroxaban versus enoxaparin after total knee arthroplasty. Lancet 2009;374:682

11. Lotke PA. Rivaroxaban for thromboprophylaxis. N Engl J Med 2008;359:2174.

12. Patel VP, Walsh $\mathbf{M}$, Sehgal B, et al. Factors associated with prolonged wound drainage after primary total hip and knee arthroplasty. J Bone Joint Surg [Am] 2007;89-A:33-8

13. Saleh K, OIson M, Resig S, et al. Predictors of wound infection in hip and knee joint replacement: results from a 20 year surveillance program. J Orthop Res 2002;20:50615.

14. No authors listed. Venous thromboembolism: reducing the risk of venous thromboembolism (deep vein thrombosis and pulmonary embolism) in inpatients undergoing surgery. NICE clinical guideline 46, 2007. http://www.nice.org.uk (date last accessed 15 March 2010)

15. Jameson SS, Bottle A, Malviya A, Muller SD, Reed MR. The impact of national guidelines for the prophylaxis of venous thromboembolism on the complications of arthroplasty of the lower limb. J Bone Joint Surg [Br] 2010;92-B:123-9.

16. Pitto RP, Hamer H, Heiss-Dunlop W, Kuehle J. Mechanical prophylaxis of deepvein thrombosis after total hip replacement: a randomised clinical trial. J Bone Joint Surg [Br] 2004;86-B:639-42

17. Maderazo EG, Judson S, Pasternak H. Late infections of total joint prostheses: a review and recommendations for prevention. Clin Orthop 1988;229:131-42.

18. Eriksson BI, Kakkar AK, Turpie AG, et al. Oral rivaroxaban for the prevention of symptomatic venous thromboembolism after elective hip and knee replacement. $J$ Bone Joint Surg [Br] 2009;91-B:636-44

19. Clayton RA, Gaston P, Howie CR. Oral rivaroxaban for the prevention of symptomatic venous thromboembolism after elective hip and knee replacement. J Bone Joint Surg [Br] 2010;92-B:468.

20. Treasure T, Chong LY, Sharpin C, et al. Developing guidelines for venous thromboembolism for The National Institute for Clinical Excellence: involvement of the orthopaedic surgical panel. J Bone Joint Surg [Br] 2010;92-B:611-16.

21. Bryne AM, Morris S, McCarthy T, Quinlan W, O'Byrne JM. Outcome following deep wond contamination in cemented arthroplasty. Int Orthop 2006;31:27-31.

22. Fender D, Harper WM, Gregg PJ. Outcome of Charnley total hip replacement across a single health region in England: the results at five years from a regional hip register. J Bone Joint Surg [Br] 1999;81-B:577-81.

23. Lehtimäki MY, Kautiainen H, Lehto UK, Hämäläinen MM. Charnley low-friction arthroplasty in rheumatoid patients: a survival study up to 20 years. J Arthroplasty 1999;14:657-61.

24. Wolowacz SE, Roskell NS, Maciver F, et al. Economic evaluation of dabigatran etexilate for the prevention of venous thromboembolism after total knee and hip replacement surgery. Clin Ther 2009;31:194-212

25. McCullagh L, Tilson L, Walsh C, Barry M. A cost-effectiveness model comparing rivaroxaban and dabigatran etexilate with enoxaparin sodium as thromboprophylaxis after total hip and total knee replacement in the Irish healthcare setting. Pharmacoeconomics 2009;27:829-46.

26. Planès $\mathbf{A}$, Samama $\mathbf{M} \mathbf{M}$, Lensing $\mathbf{A W}$, et al. Prevention of deep vein thrombosis after hip replacement: comparison between two low-molecular heparins, tinzaparin and enoxaparin. Thromb Haemost 1999;81:22-5.

27. Cusick LA, Beverland DE. The incidence of fatal pulmonary embolism after primary hip and knee replacement in a consecutive series of 4253 patients. J Bone Joint Surg [Br]2009;91-B:645-8. 